\title{
A approachable model of Wind power generation in Bhubaneswar, Odisha, India
}

\author{
Mousumi Mishra ${ }^{1}$, Sarmistha Priyambada Mishra ${ }^{2}$, Akshay Kumar \\ Asst. Prof., School of Electrical Engineering, KIIT University, Bhubaneswar, India ${ }^{1}$ \\ Student, M.Tech,4th Sem, School of Electrical Engineering, KIIT University, Bhubaneswar, India ${ }^{2,3}$
}

\begin{abstract}
This paper describes an analytical method of power generation from Wind energy in a specific location. The specific location is chosen as Bhubaneswar, Odisha, India. This paper also includes wind speed analysis, power and energy calculation, Probability Density function, Rate of return etc. over a period of time and conditions affecting it. The different parameters necessary for wind generation are studied and compared.
\end{abstract}

Keywords: Wind energy, wind speed analysis, Probability Density function, Rate of return.

\section{INTRODUCTION}

Wind energy is the kinetic energy associated with movement of large masses of air. It is the result of temperature, density, and pressure difference due to uneven heating of the atmosphere by the sun. It is of total. estimated that $1 \%$ of the solar radiation is converted to kinetic energy of the atmosphere. Thus, wind energy is an indirect form of solar energy. It is a renewable, widely available and distributed, eco-friendly, free from GHG emissions and can be used as an alternative to fossil fuel. Wind energy capacity has expanded rapidly to $336 \mathrm{GW}$ in June, 2014 and wind production is $4 \%$ of total worldwide electricity usage.

In India, As of 31st March 2014, the Installed capacity is $21136.3 \mathrm{MW}$ mainly across Tamil Nadu (7,253MW), Gujurat(3,093MW), Maharastra(2,976MW), Karnataka(2,113MW), Rajasthan(2,355MW), P(386MW), Andhra Pradesh(435MW), Kerela(35.1MW), Odisha(2MW), West Bengal(1.1MW) and others (3.2MW). Additionally, 6,000MW is planned in 2014. Wind energy contributes $8.5 \%$ of India's total Installed Capacity and generates $1.6 \%$ of total.

\section{TABLE I}

WIND POWER SCENARIO

\begin{tabular}{|c|c|c|c|}
\hline COUNTRY & $\begin{array}{c}\text { NEW } \\
\text { CAPACITY } \\
\text { (MN) }\end{array}$ & $\begin{array}{l}\text { WIND POWER } \\
\text { TOTAL } \\
\text { CAPACITY(MI) }\end{array}$ & $\begin{array}{c}\text { \% WORLD } \\
\text { TOTAL }\end{array}$ \\
\hline CHINA & 16,088 & 91,412 & 28.7 \\
\hline US & 1,084 & 61,091 & 19.2 \\
\hline GERMANY & 3,238 & 34,250 & 10.8 \\
\hline SPAIN & 175 & 22,959 & 7.2 \\
\hline INDIA & 1,729 & 20,150 & 6.3 \\
\hline UK & 1,883 & 10,531 & 3.3 \\
\hline ITALY & 444 & $\mathbf{8 , 5 5 2}$ & 2.7 \\
\hline FRANCE & 631 & 8,254 & 2.6 \\
\hline CANADA & 1,599 & 7,803 & 2.5 \\
\hline DENMARK & 657 & 4,772 & 1.5 \\
\hline REST OF & 7,761 & 48,332 & 15.2 \\
\hline WORLD & $\mathbf{3 5 , 2 8 9 M W}$ & $\mathbf{3 , 1 8 , 1 0 5 M W}$ & $\mathbf{1 0 0} \%$ \\
\hline TOTAL & \multicolumn{2}{|c}{} \\
\hline
\end{tabular}

(Fig. Wind power scenario of the World)

In India, As of 31st March 2014, the Installed capacity is $21136.3 \mathrm{MW}$ mainly across Tamil Nadu (7,253MW), Maharastra(2,976MW), Rajasthan(2,355MW), Gujurat(3,093MW), MP(386MW), Andhra Pradesh(435MW), Kerela(35.1MW), Odisha(2MW), West
Bengal(1.1MW) and others (3.2MW). Additionally, $6,000 \mathrm{MW}$ is planned in 2014. Wind energy contributes $8.5 \%$ of India's total Installed Capacity and generates $1.6 \%$

Odisha being a coastal state has higher potential for wind energy nearly $1600 \mathrm{MW}$ but it is producing $2 \mathrm{MW}$. It may be due to higher thermal reserve and is a power surplus state. Hence, a model is proposed suitable for Odisha.

\section{A. NATURE OF WIND}

\section{LITERATURE SURVEY}

It is required to understand the nature of winds to predict performance of wind turbine and proper design of supporting structure. For power generation, the speed required is between $5-25 \mathrm{~m} / \mathrm{sec}$ for $70-80 \%$ of time.

BEAUFORT NUMBER: This scale provides a wind speed classification(0-12).

VARIABILITY: It is classified into Spatial and Temporal variation.

SPATIAL: It includes climatic regions, physical geography, type of vegetations etc.

TEMPORAL: It includes geographical variation, long term wind speed variation, annual and seasonal variations and synoptic \& diurnal variation, turbulence and extreme wind speeds.

SOLIDITY: It is the ratio of the projected area of the rotor blades on the rotor plane to the swept area of the rotor.

REYNOLD'S NUMBER: It indicates the nature of flow around a body. It is the ratio between Inertia force and Viscous force. Laminar flow occurs when Reynold's no is low and viscous force are dominant and is characterised by smooth, constant and fluid motion. Turbulent flow occurs when Inertia force is dominant. In this case, eddies are formed.

\section{B. CLASSIFICATION OF WIND TURBINES}

Wind turbines are classified into two categories according to the orientation of the axis of rotation w.r.t the direction of wind i.e Vertical axis wind turbine(VAWT) \& Horizontal axis wind turbine(HAWT).In VAWT, the axis of rotation of wind turbine is perpendicular to the wind. They are used in small scale installations. In HAWT, the 
axis of rotation of these turbines is parallel to the wind. and no need for synchronisation. It is mechanically simple, These are the modern and mostly used. They are mounted highly efficient and requires low maintenance.

on towers so to raise the wind turbine above the ground for stronger winds in order to harness more energy.

\section{TYPES OF ROTORS}

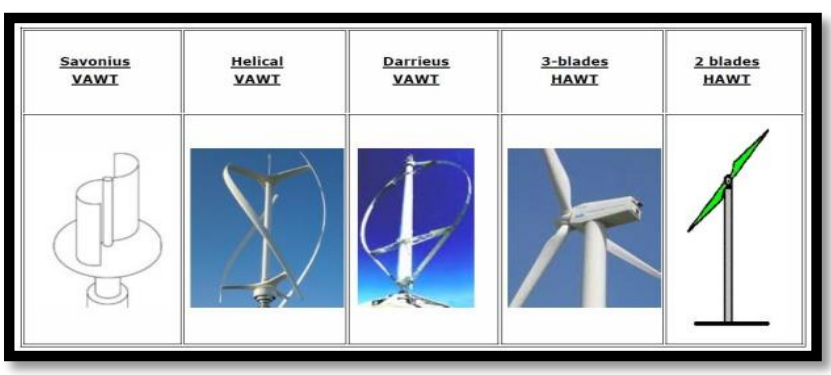

D. UPWIND AND DOWNWIND $M / C$

UPWIND M/C: In this case, rotor is located in-front of the tower. It produces higher power as it eliminates tower shadow on blades and results in lower noise, lower blade fatigue and smoother power output.

DOWNWIND M/C: The rotor is located behind the tower. It allows use of free yaw system

E. ENERGY ESTIMATION \& POWER OUTPUT FROM WIND

The Power estimated is the kinetic energy in per unit time and is given by $\mathbf{P o}=\mathbf{0 . 5} * \mathbf{p}^{*} \mathbf{A} *(\mathbf{u o} \wedge \mathbf{3})$ where $\mathrm{Po}=$ Power available in wind per unit area, $\mathrm{p}=$ density of the air, $\mathrm{A}=$ area and $\mathrm{uo}=$ Speed of free wind in unperturbed state considering no turbulence. The power output of wind turbine is given by $\mathbf{P o}=\mathbf{0 . 5} * \mathbf{p}^{*} \mathbf{A} *\left(\mathbf{u o} \mathbf{o}^{\wedge}\right) * \mathbf{C p}$, where $\mathrm{Cp}=$ power coefficient.

\section{F. POWER COEFFICIENT $(\mathrm{Cp})$}

The power captured by the wind depends on the upstream and downstream velocity of air, rotational speed of turbine and blade pitch. The theoretical maximum value of $\mathrm{Cp}$ is $16 / 27(0.593)$ called the Betz limit given by a German physicist Albert Betz. It is a function of tip speed ratio and blade pitch angle which depends on type and operating condition of the turbine. This relationship is usually provided by the turbine manufacturer in the form of a set of non dimensional curves.

G. TIP SPEED RATIO

It is defined as the ratio between speed of the tip of the rotor blade to the speed of incoming air.

$$
\mathrm{TSR}=\mathrm{R} * \mathrm{w} / \mathrm{uo}
$$

where, $\mathrm{w}=$ turbine speed in $\mathrm{rad} / \mathrm{sec}$ and $\mathrm{R}=$ radius of blade. The variation of $\mathrm{Cp}$ with TSR is shown as:

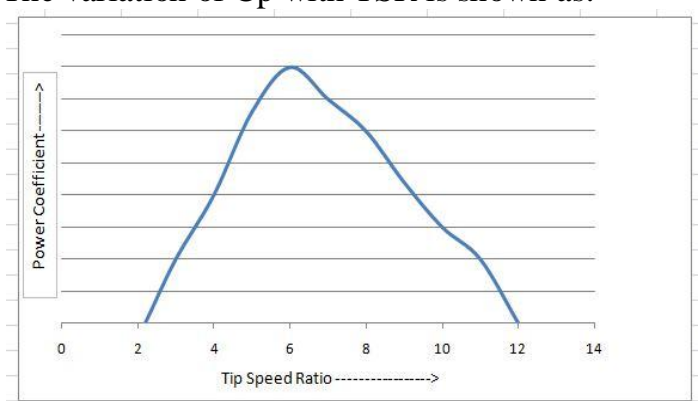

\section{H. GENERATORS USED FOR WIND ENERGY}

\section{EXTRACTION SYSTEM}

Squirrel Cage Induction Generator(SCIG): It is preferred in distributed generation as it has the ability to auto start
Permanent Magnet Synchronous Generator(PMSG): When PMSG machines are used, the wind generator doesn't need to supply excitation system and are connected to grid and are widely used in variable speed wind generators for their high efficiency and higher generated power to weight ratio than induction generators. They have a loss free rotor and the power losses are mainly related to the stator windings and the stator core.

Doubly fed Induction Generator(DFIG): Wound rotor induction generators(WRIG) are provided with three phase windings on the stator and on the rotor. The stator is directly connected to the grid while the rotor winding is connected via slip rings to the grid through a converterinverter combination. WRIG provides constant (regulated) voltage and frequency in the stator terminals. The rotor is supplied through a static power converter at a variable voltage and a variable frequency as per the speed variation.

\section{WIND SPEED PREDICTION AND FORECASTING}

STATISTICAL METHODS

Persistent Forecast:

$\underline{\mathbf{Y}} \mathbf{k}=\mathbf{Y}(\mathbf{k}-1)$

nth Order Autoregressive model:

$\underline{\mathbf{Y}} k=\Sigma$ aiY(k-i); $i=1, \ldots . . n$

nth Order Autoregressive mth order Moving Average model:(ARMA(n,m))

\section{$\underline{Y} \mathbf{~} k=\Sigma$ aiY(k-i) $+\Sigma b j e(k-j) ; i=1, \ldots n ; j=1, \ldots m$ where, $\mathbf{e k}=\mathbf{Y} \mathbf{k}=\mathbf{Y}(\mathbf{k})$}

\section{ARMAX model:}

In addition to ARMA model, an exogenous variable ' $\mathrm{X}$ ' is included which influences Ý.

Model parameters ai \& bj can be estimated in various ways like Method of Recursive Least Squares(RLS).

\section{METEOROLOGICAL METHODS}

It uses detailed analysis and modelling of the atmosphere.

\section{J. WIND VELOCITY DISTRIBUTION}

The wind regime is influenced by regional and local effects and depends on seasonal and short-time variations. Wind velocity varies with height above ground and influenced by surface roughness. Assuming stable conditions, the dependence of velocity ' $\mathrm{V}$ ' on height ' $\mathrm{Z}$ ' is given as

$\mathrm{V} 2(\mathrm{Z} 2)=\mathrm{V} 1 *\{\ln (\mathrm{Z} 2 / \mathrm{Z} 0) / \ln (\mathrm{Z} 1 / \mathrm{Z} 0)\}$
where $\mathrm{Z} 0=$ Roughness length;
$\mathrm{Z} 0=0.03 \mathrm{~m}$ for farmland
$=0.1 \mathrm{~m}$ for scattered shrubs \& trees
$=0.5-1.6 \mathrm{~m}$ for forests.

This equation can be used when calculating the reference energy used in the project stage.

WEIBULL DISTRIBUTION:

It includes probability density function(PDF) which is defined by

$\mathrm{f}(\mathrm{x} ; \lambda, \mathrm{K})=\mathrm{K} / \lambda^{*}(\mathrm{x} / \lambda)^{\wedge}\left\{\mathrm{e}^{\wedge}\left((-\mathrm{x} / \lambda)^{\wedge} \mathrm{K}\right)\right\} ; \mathrm{x}>=0$ $=0 ; \mathrm{x}<0$

The PDF of a continuous random variable is a function that describes the relative likelihood for this random 
variable to take on a given value. The probability of a random variable falling within a particular range of values is given by the integral of this variable density over that range.

$\operatorname{Pr}[\mathrm{a}<\mathrm{X}<\mathrm{b}]=\int \mathrm{f}(\mathrm{x}) \cdot \mathrm{dx} ; \mathrm{a}<\mathrm{x}<\mathrm{b}=\mathrm{F}(\mathrm{x})$;

$\lambda, \mathrm{K}=$ Parameters which affects the distribution.

$\mathrm{K}=$ Shape Parameters which affects the shape of distribution rather than simply shifting it.

$\lambda=$ Scale Parameters which stretches or shrinks the distribution.

$\mathrm{K}=1$ (Weibull Distribution);K=2(Rayleigh Distribution)

RAYLEIGH DISTRIBUTION:

It represents wind speed distribution .

$\mathrm{f}(\mathrm{x} ; \lambda, \mathrm{K})=2 / \lambda^{*}(\mathrm{x} / \lambda)^{\wedge}\left\{\mathrm{e}^{\wedge}\left((-\mathrm{x} / \lambda)^{\wedge} 2\right)\right\} ; \mathrm{x}>=0$ $=0 ; \mathrm{x}<0$

Wind Speed distribution improves estimation of Wind Power Potential.

\section{SYSTEM CONFIGURATION}

LOCATION: BHUBANESWAR,ODISHA,INDIA

ELEVATION:46m

LATITUDE: $20.2 \mathrm{deg}$; LONGITUDE: $85.8 \mathrm{deg}$

AREA $=5026 \mathrm{~m} 2$

\section{A. NATURE OF WIND}

The wind speed of Bhubaneswar for the year of 2014 varies from 0.278 to $13.07 \mathrm{~m} / \mathrm{sec}$. Its Beaufort No varies from 0 to 6 which indicates calm to strong wind.

\section{B. TYPE OF TURBINE USED}

A HAWT is considered having 3 blades rotor. An upwind machine is considered as it produces higher power as it eliminates tower shadow on blades and results in lower noise, lower blade fatigue and smoother power output.

C. ENERGY ESTIMATION \& POWER OUTPUT FROM WIND

Yearly data of wind speed is taken for 2013 and its power output and energy is calculated.

\begin{tabular}{|c|c|c|c|c|c|c|c|c|c|}
\hline MONIH & DAY & $\begin{array}{l}\text { WIND } \\
\text { SPEED }\end{array}$ & PRESSURE & TEMP & TEMP & DENSITY & $\begin{array}{l}\text { POWER } \\
\text { IAREA }\end{array}$ & POWER & ENRRC \\
\hline & & (m/sec) & $(\mathrm{Pa})$ & $\left({ }^{\circ} \mathrm{C}\right)$ & ("K) & (b) & (Watttm2) & (KW) & (KWh) \\
\hline \multicolumn{10}{|l|}{ JAN } \\
\hline & 1 & 1.112 & 101309 & 23 & 377,309 & 1.193 & \begin{tabular}{|l|} 
\\
\end{tabular} & 4 4.1207975 & 98.899139 \\
\hline & 2 & 1.946 & 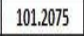 & 24 & 374.2075 & 1.187 & 4.3749477 & 21.988487 & 527,72369 \\
\hline & 3 & 1.668 & 101.238 & 26 & 374.238 & 1.180 & 2.7374653 & 13.7585 & 330.20401 \\
\hline & 4 & 1.788 & 101.3 & 23.33 & 374,3 & 1.191 & 3.4042737 & 17.10988 & 410.63712 \\
\hline & 5 & 0.894 & 101.475 & 23,33 & 374,475 & 1.193 & 0.4262693 & 2.14224297 & 51.418314 \\
\hline & 6 & 1.668 & 101.514 & 23 & 374.514 & 1.195 & 2.7727485 & 13.935834 & 334,46001 \\
\hline & 7 & 1.668 & 101.4107 & 24 & 374.4107 & 1.190 & 2,7606006 & 13.874779 & 332.99469 \\
\hline & 8 & 1.946 & 101.425 & 21 & 374,425 & 1.202 & 4,4290879 & 22.260596 & 534.2543 \\
\hline & 9 & 1.39 & \begin{tabular}{|l}
101.463 \\
\end{tabular} & 19 & 374,463 & 1.211 & 1.6257635 & 8.1710871 & 196.106009 \\
\hline & 10 & 0.894 & 101337 & 20.556 & 374,337 & 1.203 & 0.4297123 & 2.1597338 & 51.833612 \\
\hline & 11 & 1.39 & 101.271 & 20 & 374.271 & 1.204 & 1.6171488 & 8.1277899 & 195.06696 \\
\hline & 12 & 1.39 & 101.51228 & 20 & 374.51228 & 1.207 & 1.6210017 & 8.1471545 & 195.53171 \\
\hline & 13 & 1.112 & 101.664 & 20 & 374.664 & 1.209 & 0.8311933 & 4.1775776 & 100.26186 \\
\hline & 14 & 0.834 & 101.625 & 23 & 374,625 & 1.196 & 0.3469725 & 1.743884 & 41.853216 \\
\hline & 15 & 0.894 & 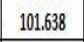 & 23.889 & 374.638 & 1.193 & 0.4261502 & 2,1418308 & 51.403938 \\
\hline & 16 & 0.834 & 101.563 & 24 & 374,563 & 1.192 & 0.345933 & 1.736552 & 41.686848 \\
\hline & 17 & 0.834 & 101.538 & 24 & 374,538 & 1.191 & 0.345082 & 1.7365244 & 41.676586 \\
\hline
\end{tabular}

\begin{tabular}{|c|c|c|c|c|c|c|c|c|c|}
\hline & 18 & 1.112 & 101.712 & 24 & 374.712 & 1.193 & 0.820386 & 4.1232598 & | 98.958235 \\
\hline & 19 & 0.834 & 101.775 & 24 & 374.775 & 1.194 & 0.3463147 & 1.700577 & $41.773864 \mid$ \\
\hline & 20 & 1.112 & 101.737 & 22 & 374.737 & 1.202 & 0.8261509 & 4.1522344 $\mid$ & 99.653626 \\
\hline & 21 & 1.668 & 101.638 & 22 & 374.638 & 1.200 & 2.785546 & 14,000154 & 336.00371 \\
\hline & 22 & 1.39 & 101.663 & 21 & 374,663 & 1.205 & 1.6178867 & 8.1349984 & 195.15596 \\
\hline & 23 & 0.834 & 101.675 & 20 & 374,675 & 1.209 & 0.3506976 & 1.7626062 & 4230255 \\
\hline & 24 & 1.39 & 101.65 & 22 & 374.65 & 1.201 & 1.62121961 & 8.1028978 & 194.46955 \\
\hline & 25 & 1.39 & 101.588 & 20 & 374.588 & 1.208 & 1.6222108 & 8.1523217 & 195.67756 \\
\hline & 26 & 1.668 & 101.5 & 20 & 374.5 & 1.207 & 28007521 & 14.07658 & 337.87792 \\
\hline & 27 & 1.112 & 101.557 & 21 & 374.557 & 1.204 & 0.8274943 & 4.1589863 & 99.81567 \\
\hline & 28 & 1.668 & 101.633 & 20 & 374.633 & 1.209 & 2.804422 & 14.095025 & 338.2806 \\
\hline & 29 & 1.112 & 101.5 & 20 & 374.5 & 1.207 & 0.8298525 & 4.1708385 & 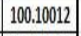 \\
\hline & 30 & 0.834 & 101.6 & 22 & 374.6 & 1.200 & 0.3480631 & 1.799365 & 41.98476 \\
\hline & 31 & 0.834 & 101.6 & 22 & 374.6 & 1.200 & 0.3480631 & 1.749365 & 41.98476 \\
\hline \multicolumn{10}{|l|}{ FLB } \\
\hline & 1 & 0.834 & 101.63 & 23 & 374.63 & 1.196 & 0.3469896 & 1.74396988 & 41.855275 \\
\hline & 2 & 1.112 & 101.55 & 22 & 374.55 & 1.199 & 0.8246324 & 4.1446023 & 99.470456 \\
\hline & 3 & 1.39 & 101.44 & 24 & 374,44 & 1.190 & 1.5980314 & 8.03177056 & 192.76094 \\
\hline & 4 & 0.834 & 101.59 & 24 & 374.59 & 1.192 & 0.3456852 & 1.73744138 & 41.69793 \\
\hline & 5 & 0.834 & 101.65 & 25 & 374,65 & 1.189 & 0.3477286 & 1.7326062 & 41.582549 \\
\hline & 6 & 0.834 & 101.5 & 25 & 374.5 & 1.187 & 0.34422 & $1.7300495 \mid$ & 41.521187 \\
\hline & 7 & 0.556 & 101.24 & 26 & 374.24 & 1.180 & 0.10101896 & 0.5095842 & 12.23002 \\
\hline & 8 & 1.668 & 101.3 & 24 & 374.3 & 1.188 & 2.77575871 & $13.859633 \mid$ & $332.63119 \mid$ \\
\hline & 9 & 1.668 & 101.39 & 24 & 374,39 & 1.189 & 2.7600371 & 13.871946 & 332.92672 \\
\hline & 10 & 1.79 & 101.41 & 23.33 & 374,41 & 1.192 & 3.4.194193 & 17.186002 & 412.,46404 \\
\hline & 11 & 0.834 & 101.39 & 23 & 374,39 & 1.193 & 0.34461702 & 1.7398514 & 41.756433 \\
\hline & 12 & 0.834 & 101.39 & 22 & 374,39 & 1.198 & 0.3473437 & 1.7457492 & 41.897981 \\
\hline & 13 & 0.834 & 101.26 & 24 & 374.26 & 1.188 & 0.345623 & 1.73177 & 41.562481 \\
\hline & 14 & 1.34 & 101.28 & 25 & 374,28 & 1.184 & 1,4246557 & 7.1603197 & 171.84767 \\
\hline & 15 & 1.668 & 101.24 & 26 & 374.24 & 1.180 & 2.7375193 & 13.758772 & 330.21053 \\
\hline & 16 & 1.668 & 101.14 & 25 & 374.14 & 1.183 & 2.7439926 & 13.791307 & 330.99136 \\
\hline & 17 & 1.668 & 101 & 23 & 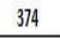 & 1.189 & 2,7587091 & 13.865272 & 332.76653 \\
\hline & 18 & 1.668 & 101.21 & 24 & 374.21 & 1.187 & 2.7551371 & 13.847319 & 332.33566 \\
\hline & 19 & 1,39 & 101.38 & 22 & 374,38 & 1.197 & 1.6079139 & 8.0813751 & 193.953 \\
\hline & 20 & 1.39 & 10138 & 24 & 374,38 & 1.189 & 1.5970862 & 8.026955 & 192.64692 \\
\hline & 21 & 1.112 & 101.41 & 24 & 374,41 & 1.190 & 0.8179501 & 4.1.1110171 & 98.6644111 \\
\hline & 22 & 0.834 & 101.53 & 24 & 374.53 & 1.191 & \begin{tabular}{|l|l|} 
\\
\end{tabular} & 1.7763876 & 41.673303 \\
\hline & 23 & 1.668 & 101.45 & 25 & 374,45 & 1.186 & 2.7524031 & 13.833578 & 332.0587 \\
\hline & 24 & 1.39 & 101.4 & 26 & 4 & 1.182 & 1.5867163 & 7.974836 & 191.39606 \\
\hline & 25 & 1.668 & 101.43 & 26 & 374,43 & 1.182 & 2.7426569 & 13.784594 & 330.83025 \\
\hline & 26 & 1.112 & 101.43 & 26 & 374,43 & 1.182 & 0.8126391 & 4.084324 & 98.023777 \\
\hline & 27 & 1.112 & 101.3 & 26 & 4.3 & 1.180 & 0.8115975 & 4.0790893 & 97.898143 \\
\hline & 28 & 1.112 & 101.12 & 28 & 374,12 & 1.171 & 0.804722 & 4.0447857 & 97.074857 \\
\hline \multicolumn{10}{|l|}{ MAR } \\
\hline & 1 & 1.39 & 101.24 & 27 & 374,24 & 1.176 & 1.5789319 & \begin{tabular}{|l}
.9357116 \\
\end{tabular} & 190,45708 \\
\hline & 2 & 1.67 & 101.37 & 26 & 374,37 & 1.181 & 2.7509062 & 13.86054 & 331.82531 \\
\hline
\end{tabular}


INTERNATIONAL JOURNAL OF INNOVATIVE RESEARCH IN ELECTRICAL, ELECTRONICS, INSTRUMENTATION AND CONTROL ENGINEERING Vol. 3, Issue 2, February 2015

\begin{tabular}{|c|c|c|c|c|c|c|c|c|}
\hline 3 & 1.11 & 101.34 & 26 & 374.34 & 1.181 & 0.807545 & 4.0587214 & 97.40! \\
\hline 4 & 1.67 & 101.43 & 26 & 374,43 & 1.182 & 2.7525344 & 13.834238 & 3320. \\
\hline 5 & 1.39 & 101.46 & 27 & 374,46 & 1.178 & 1.582363 & 7.9529563 & 190.8 \\
\hline 6 & 1.39 & 101.43 & 28 & 374,43 & 1.174 & 1.5766396 & 7.9241900 & 190.11 \\
\hline 7 & 2.22 & 101.37 & 28 & 374.37 & 1.173 & 6.4193341 & 32.263573 & 774.3: \\
\hline 8 & 1.95 & 101.31 & 28 & 374.31 & 1.173 & 4.3478821 & 21.852456 & 524.4. \\
\hline 9 & 2.22 & 101.11 & 28 & 374.11 & 1.170 & 6.4028694 & 32.180822 & 772.3 \\
\hline 10 & 2.5 & 100.99 & 30 & 373.99 & $\begin{array}{l}1.161 \\
\end{array}$ & 9.0728531 & 45.600159 & 1094.، \\
\hline 11 & 3.34 & 101.01 & 30 & 374,01 & 1.162 & 21.639601 & 108.76064 & 2610. \\
\hline 12 & 3.61 & 100.95 & 30 & 373.95 & $\begin{array}{l}1.161 \\
\end{array}$ & 27,306963 & $\begin{array}{l}137.2448 \\
\end{array}$ & 3293.1. \\
\hline 13 & 1.95 & 101.01 & 30 & 374,01 & 1.162 & 4.3063932 & 21.643932 & 519.4: \\
\hline 14 & 2.22 & 101.14 & 30 & 374.14 & 1.163 & 6.3624935 & 31.977892 & 767.41 \\
\hline 15 & 1.39 & 101.24 & 30 & 374.24 & 1.164 & 1.5632989 & 7.8571402 & 188.5 \\
\hline 16 & 1.34 & 101.34 & 28.89 & 374,34 & 1.170 & 1.4071315 & 7.07242428 & 169.7: \\
\hline 17 & 1.67 & 101.26 & 29 & 374.26 & 1.168 & 2.7206239 & 13.673855 & 328.1. \\
\hline 18 & 1.39 & 100.99 & 28 & 373.99 & 1.169 & 1.5698002 & 7.8898159 & 189,3! \\
\hline 19 & 2.22 & 100.95 & 30 & 373.95 & 1.161 & 6.350541 & 31.917819 & 766.04 \\
\hline 20 & 1.67 & 100.96 & 30 & 373.96 & 1.161 & 2.7036112 & 13.58835 & 326.1 \\
\hline 21 & 2.69 & 100.84 & 30 & 373.84 & 1.160 & 11.285873 & 56.722797 & 1361: \\
\hline 22 & 4.03 & 100.67 & 31.11 & 373.67 & 1.153 & 37,746146 & 189.71213 & 4553.1. \\
\hline 23 & 3.61 & 100.86 & 31 & 373.86 & 1.156 & 27.192873 & 136.67138 & 3280: \\
\hline 24 & 3.34 & 100.95 & 30 & 373.95 & $\begin{array}{l}1.161 \\
\end{array}$ & 21.626747 & 108.696003 & 2608: \\
\hline 25 & 3.06 & 100.69 & 32 & 373.69 & 1.150 & 16.479305 & 82.824986 & 1987: \\
\hline
\end{tabular}

\begin{tabular}{|c|c|c|c|c|c|c|c|c|c|}
\hline & 26 & 2.22 & 100.59 & 32 & 373.59 & 1.149 & \begin{tabular}{|c|c|c|}
6 & 3 \\
\end{tabular} & \begin{tabular}{|l|l|}
31.595446 & 75 \\
\end{tabular} & 758.29069 \\
\hline & 27 & 1.95 & 100.73 & 31 & 373.73 & 1.155 & 4.2803294 & 21.512936 & 516.31045 \\
\hline & 28 & 1.95 & 100.81 & 32 & 373.81 & 1.152 & $|4,2696838|$ & $\begin{array}{ll}21.459431 & 5\end{array}$ & 515,02634 \\
\hline & 29 & 1.95 & 100.91 & 30 & 373.91 & 1.160 & \begin{tabular}{|l|l}
43012299 \\
\end{tabular} & 21.62505 & 518.94012 \\
\hline & 30 & 3.06 & 100.66 & 32 & 373.66 & 1.150 & \begin{tabular}{|l|l|}
16.474395 & 8 \\
\end{tabular} & \begin{tabular}{|l|l|}
82.800309 & 15 \\
\end{tabular} & 1987,2074 \\
\hline & 31 & 3.34 & 1000.7 & 32 & 373.7 & 1.150 & \begin{tabular}{|l|l}
21.431726 & 1 \\
\end{tabular} & \begin{tabular}{|l|l|}
107.71585 & 25 \\
\end{tabular} & 2585.1805 \\
\hline \multicolumn{10}{|l|}{$\mathrm{APR}$} \\
\hline & 1 & 2.78 & 100.84 & 32 & 373.84 & 1.152 & \begin{tabular}{|l|l|}
12.375223 \\
\end{tabular} & \begin{tabular}{|l|l|}
62.198223 & 1 \\
\end{tabular} & 1992,7574 \\
\hline & 2 & 2.5 & 100.85 & 31 & 373.85 & 1.156 & \begin{tabular}{|l|l|}
9.030472 \\
\end{tabular} & \begin{tabular}{|l|l|}
45.387152 & 10 \\
\end{tabular} & 1089,2917 \\
\hline & 3 & 3.61 & 100.85 & 32 & 373.85 & 1.152 & \begin{tabular}{|l|l}
27,101029 & 1 \\
\end{tabular} & \begin{tabular}{|l|l|}
136.20977 \\
\end{tabular} & 3269,0345 \\
\hline & 4 & 2.5 & 100.49 & 33 & 373.49 & 1.144 & \begin{tabular}{|l|l|l|}
89394243 & 4 \\
\end{tabular} & \begin{tabular}{|l|l|l|}
44,925547 & 10 \\
\end{tabular} & 1078.3001 \\
\hline & 5 & 1.95 & 100.48 & 34 & 373.48 & 1.140 & \begin{tabular}{l|l|l} 
& 2.2279826 \\
\end{tabular} & \begin{tabular}{|l|l|}
21.249841 & 50 \\
\end{tabular} & 509.99617 \\
\hline & 6 & 2.24 & 100.57 & 33.33 & 373.57 & 1.144 & \begin{tabular}{l|l|l}
6.4285212 & 3 \\
\end{tabular} & \begin{tabular}{|l|l}
32.309748 & 77 \\
\end{tabular} & 775.43394 \\
\hline & 7 & 3.34 & 100.05 & 34 & 373.05 & 1.136 & 21,154669 & \begin{tabular}{|l|l|}
106,32336 \\
\end{tabular} & 2551.7607 \\
\hline & 8 & 3.13 & 100.6 & 33.33 & 373.6 & 1.144 & \begin{tabular}{l|l}
17.544038 & 8 \\
\end{tabular} & \begin{tabular}{|l|l|}
88.176337 & 21 \\
\end{tabular} & 2116.2321 \\
\hline & 9 & 13.07 & 100.69 & 33 & 373.69 & 1.147 & \begin{tabular}{l|l}
1279.911 & 6 \\
\end{tabular} & \begin{tabular}{|l|l|}
6432.8329 & 15 \\
\end{tabular} & 154387.99 \\
\hline & 10 & 4.73 & 100,61 & 33 & 373.61 & 1.146 & \begin{tabular}{l|l}
60.616555 & 3 \\
\end{tabular} & \begin{tabular}{|l|l}
304,65881 & 7 \\
\end{tabular} & 7311.8114 \\
\hline & 11 & 4.17 & 100.63 & 33 & 373.63 & 1.146 & \begin{tabular}{ll|l}
41,543427 & 2 \\
\end{tabular} & \begin{tabular}{|l|l|}
208.79726 & 50 \\
\end{tabular} & 5011.1343 \\
\hline & 12 & 4.73 & 100.76 & 32 & 373.76 & 1.151 & \begin{tabular}{l|l}
60.905968 & 3
\end{tabular} & \begin{tabular}{|l|l|}
306.11339 & 7 \\
\end{tabular} & 7346,7215 \\
\hline & 13 & 4.45 & 100.86 & 32 & 373.86 & 1.152 & \begin{tabular}{|c|c}
50,767674 & 2
\end{tabular} & \begin{tabular}{|l|l|}
255.15833 & 6 \\
\end{tabular} & 6123.7999 \\
\hline & 14 & 3.06 & 100.86 & 31 & 373.86 & 1.156 & \begin{tabular}{l|l}
16.561427 & 8
\end{tabular} & \begin{tabular}{|l|l|}
83.237735 & 15 \\
\end{tabular} & 1997,7056 \\
\hline & 15 & 3.34 & 100.69 & 31 & 373.69 & 1.154 & \begin{tabular}{l|l}
21.500089 & 1 \\
\end{tabular} & 108,05945 & 2593,4268 \\
\hline & 16 & 8.34 & 100.28 & 32 & 373.28 & 1.146 & 332.27736 & \begin{tabular}{|l|l|}
1670,026 & 40 \\
\end{tabular} & 40080.624 \\
\hline & 17 & 3.61 & 100.24 & 32 & 373.24 & 1.145 & 26.937106 & \begin{tabular}{|l|l|}
6 & 135.38589 \\
\end{tabular} & 3249.2614 \\
\hline & 18 & 4.73 & 100.33 & 33 & 373.33 & 1.142 & 60.447858 & \begin{tabular}{|l|l|}
803.81093 \\
\end{tabular} & \begin{tabular}{|l|l|}
7291.4624 \\
\end{tabular} \\
\hline & 19 & 4.47 & 100.57 & 31.11 & 373.57 & 1.152 & 51.45747 & 258.62524 & 46207.058 \\
\hline & 20 & 2.78 & 100.84 & 26 & 373.84 & 1.175 & 12.623627 & $\begin{array}{ll}7 & 63.446348 \\
\end{array}$ & \begin{tabular}{|l|l|l}
8522.7123 \\
\end{tabular} \\
\hline & 21 & 1.95 & 100.94 & 27 & 373.94 & 1.172 & 4.346443 & 21.845222 & 2524.28534 \\
\hline & 22 & 1.39 & 100.99 & 27 & 373.99 & 1.173 & 1.5750329 & $\begin{array}{ll}9 & 7.9161153 \\
\end{array}$ & \begin{tabular}{|l|l|}
389.98677 \\
\end{tabular} \\
\hline & 23 & 2.22 & 101.05 & 30 & 374,05 & 1.162 & 6.3568318 & \begin{tabular}{|l|l|}
81.999437 \\
\end{tabular} & 7666.78648 \\
\hline & 24 & 1.95 & 101.03 & 31 & 374,03 & 1.158 & 4.2930773 & $\begin{array}{lll}3 & 21.577007 \\
\end{array}$ & 517.84816 \\
\hline & 25 & 3.06 & 100.95 & 32 & 373.95 & 1.153 & 16.521857 & \begin{tabular}{l|l|}
7 & 83.038856 \\
\end{tabular} & \begin{tabular}{|l|l}
6 & 1992.9325 \\
\end{tabular} \\
\hline & 26 & 2.78 & 100.88 & 32 & 373.88 & 1.152 & 12,380202 & \begin{tabular}{|l|l|}
2 & 62.222895 \\
\end{tabular} & \begin{tabular}{|l|l|l}
1493,3495 \\
\end{tabular} \\
\hline & 27 & 3.89 & 100.69 & 32 & 373.69 & 1.150 & 33.855046 & $\begin{array}{lll}6 & 170.15546 \\
\end{array}$ & \begin{tabular}{|l|l|}
6 & 4083.7311 \\
\end{tabular} \\
\hline & 28 & 5.01 & 100.49 & 34 & 377.49 & 1.141 & 71,710996 & \begin{tabular}{l|l|}
6 & 360.41947 \\
\end{tabular} & 88550.0672 \\
\hline & 29 & 5.56 & 100.56 & 34 & 373.56 & 1.141 & 98,084272 & \begin{tabular}{|l|l|}
292.97155 \\
\end{tabular} & 11831.317 \\
\hline & 30 & 5.01 & 100.51 & 34 & 373.51 & 1.141 & 71.725269 & \begin{tabular}{|l|l|}
9 & 360.4912 \\
\end{tabular} & 8651.7888 \\
\hline \multicolumn{10}{|l|}{ MAY } \\
\hline & 1 & 5.004 & 100.36 & 34 & 373.36 & 1.139 & 71.361224 & \begin{tabular}{|l|l|l|}
4 & 358.66151 \\
\end{tabular} & 8607.8762 \\
\hline & 2 & 5.282 & 100.18 & 34 & 373.18 & 1.137 & 83.777221 & 1421.06431 & 101005.544 \\
\hline & 3 & 6.116 & 100.14 & 34 & 373.14 & 1.137 & 130,00491 & $\begin{array}{lll}1 & 653.40467 \\
\end{array}$ & 15681.712 \\
\hline & 4 & 7.23 & 100.25 & 34 & 373.25 & 1.138 & 215.00522 & \begin{tabular}{|l|l|} 
& 1080,6162 \\
\end{tabular} & 25934.79 \\
\hline & 5 & 7.51 & 100.48 & 33 & 373.48 & 1.144 & 242.30709 & \begin{tabular}{l|l|}
9 & 1217.8354 \\
\end{tabular} & $4 \quad 29228.05$ \\
\hline & 6 & 7.51 & 100.44 & 34 & 373.44 & 1.140 & 241.42167 & $\begin{array}{lll}7 & 1213.3853 \\
\end{array}$ & \begin{tabular}{|l|l|} 
& 29121.247 \\
\end{tabular} \\
\hline & 7 & 5.84 & 100.43 & 30 & 373.43 & 1.155 & 115.01315 & \begin{tabular}{|l|l|l}
5 & 578,05007 \\
\end{tabular} & \begin{tabular}{|l|l|}
13873,346 \\
\end{tabular} \\
\hline & 8 & 6.39 & $100 . .28$ & 32 & 373.28 & 1.146 & $\mid$ 149.45318 & \begin{tabular}{l|l|}
8 & 751.1517
\end{tabular} & 18007.641 \\
\hline
\end{tabular}


INTERNATIONAL JOURNAL OF INNOVATIVE RESEARCH IN ELECTRICAL, ELECTRONICS, INSTRUMENTATION AND CONTROL ENGINEERING Vol. 3, Issue 2, February 2015

\begin{tabular}{|c|c|c|c|c|c|c|c|c|c|}
\hline & 9 & 6.116 & 100.26 & 34 & 373.26 & 1.138 & $\mid 130,1607$ & |654.18766 & 15700.504 \\
\hline & 10 & 6.116 & 100.23 & 34 & 373.23 & 1.138 & 130.12175 & 653.99191 & 15695.806 \\
\hline & 11 & 5.282 & 100.11 & 34 & 373.11 & 1.136 & 83.718682 & 420,7701 & 10098.482 \\
\hline & 12 & 5.282 & 10036 & 32 & 373,36 & 1.147 & 84.478095 & 424.58691 & 10190.086 \\
\hline & 13 & 2.224 & 10039 & 28 & 373.39 & 1.162 & 6.3917005 & 32.124687 & 770.99248 \\
\hline & 14 & 1.34 & 100.23 & 31.11 & 373.23 & 1.148 & 1.3815593 & 6.9437171 & 166.64921 \\
\hline & 15 & 1.67 & 100.1 & 30 & 373,1 & 1.151 & 2.6805812 & 13.4726001 & 323,34243 \\
\hline & 16 & 2.5 & 100.1 & 31 & 373.1 & 1.147 & 8.9633143 & 45,049618 & 1081.1908 \\
\hline & 17 & 4.73 & 100.26 & 32 & 373.26 & 1.145 & 60.603735 & 304.59437 & 7310.2649 \\
\hline & 18 & 6.39 & 100.3 & 34 & 373.3 & 1.138 & \begin{tabular}{|l|l|}
148.50916 \\
\end{tabular} & 746.40703 & 17913,769 \\
\hline & 19 & 6.116 & 100.19 & 33 & 373.19 & 1.141 & 130.49488 & 655.86729 & 15740.815 \\
\hline & 20 & 5.84 & 100.05 & 35 & 373.05 & 1.132 & 112.71794 & 566.52034 & 13596,488 \\
\hline & 21 & 6.39 & 100.14 & 33 & 373.14 & 1.140 & \begin{tabular}{|l|l|}
148.75681 \\
\end{tabular} & 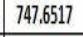 & 17943.641 \\
\hline & 22 & 3.58 & 99.99 & 35 & 372.99 & 1.131 & 25.950339 & 130.4264 & 3130.2337 \\
\hline & 23 & 4.45 & 99.93 & 34 & 372.93 & 1.134 & 49.971876 & 251.15865 & 6027.8076 \\
\hline & 24 & 4.45 & 99.89 & 34 & 372.89 & 1.134 & 49.951873 & 251.05811 & 60253947 \\
\hline & 25 & 2.78 & 99.85 & 35 & 372.85 & 1.130 & 12.134443 & 60.987711 & 1463.7051 \\
\hline & 26 & 2.22 & 100 & 32 & 373 & 1.142 & 6.2455276 & 31.410126 & 753.84302 \\
\hline & 27 & 0.834 & 100.13 & 28 & 373.13 & 1.159 & 0,3361894 & 1.6896878 & 40.552507 \\
\hline & 28 & 1.34 & 100.06 & 31.11 & 373,06 & 1.146 & 13379216 & 6.9319398 & 166.36656 \\
\hline & 29 & 2.5 & 99.98 & 27 & 372.98 & 1.161 & 9.0719367 & 45.595554 & 1094,2933 \\
\hline & 30 & 3.06 & 99.76 & 30 & 372.76 & 1.147 & 16.434867 & 82.601643 & 1982.4394 \\
\hline & 31 & 2.78 & 100 & 29 & 373 & 1.154 & 12.394116 & 62.292826 & \begin{tabular}{|l|l|} 
& 1495.0278 \\
\end{tabular} \\
\hline \multicolumn{10}{|l|}{$\mathrm{JUNL}$} \\
\hline & 1 & 4.45 & 100.31 & 31 & 373.31 & 1.150 & 50.656921 & 254.60168 & 6110.4040 \\
\hline & 2 & 3.06 & 100.28 & 30 & 373.28 & 1.153 & 16.520534 & 83.032204 & \begin{tabular}{|l|}
1992.7729 \\
\end{tabular} \\
\hline & 3 & 2.78 & 100.24 & 32 & 373.24 & 1.145 & 12.30166 & 61.828142 & \begin{tabular}{|l|l|}
1483.8754 \\
\end{tabular} \\
\hline & 4 & 1.95 & 100.31 & 31 & 373.31 & 1.150 & $\begin{array}{l}4.2624823 \\
\end{array}$ & 21.423236 & 514.15766 \\
\hline & 5 & 2.5 & 100.26 & 32 & 373.26 & 1.145 & 8.9482064 & 44.973686 & 1079.3655 \\
\hline & 6 & 2.78 & 100.18 & 32 & 373.18 & 1.144 & 12.294297 & 61.791134 & 1482.9872 \\
\hline & 7 & 2.22 & 100.11 & 32 & 373.11 & 1.144 & 6.2564021 & 31.444677 & 754,67225 \\
\hline & 8 & 2.5 & 100.03 & 29 & 373.03 & 1.154 & 9.0163645 & 45.3116248 & 1087,5899 \\
\hline & 9 & 1.95 & 99.93 & 31 & 372.93 & 1.145 & 4.2463349 & 21.342079 & \begin{tabular}{|l|}
512.2099 \\
\end{tabular} \\
\hline & 10 & 2.22 & 99.89 & 30 & 372.89 & 1.149 & 6.2838588 & 31.582674 & \begin{tabular}{|l|l|}
757,98418 \\
\end{tabular} \\
\hline & 11 & 0.834 & 99.91 & 30 & 372.91 & 1.149 & \begin{tabular}{|l|}
0.3332365 \\
\end{tabular} & 1.6748467 & 40.196322 \\
\hline & 12 & 2.22 & 99.53 & 26 & 372.53 & 1.160 & \begin{tabular}{|l|l|} 
\\
\end{tabular} & 31.889839 & 765.35614 \\
\hline & 13 & 3.06 & 99.39 & 26 & 372.39 & 1.158 & \begin{tabular}{|l|}
16.592961 \\
\end{tabular} & 83,396221 & 2001.5093 \\
\hline & 14 & 2.22 & 99.48 & 28 & 372.48 & 1.152 & \begin{tabular}{|l|}
6.2996484 \\
\end{tabular} & 31.662033 & \begin{tabular}{|l|}
759.88879 \\
\end{tabular} \\
\hline & 15 & 3.34 & 99.81 & 28 & 372.81 & 1.155 & 21.524599 & \begin{tabular}{|l|}
108.18263 \\
\end{tabular} & 2596.3832 \\
\hline & 16 & 3.133 & 100.09 & 30 & 373.09 & 1.151 & 17.697721 & 88.948748 & 2134,7699 \\
\hline & 17 & 3.06 & 100.13 & 30 & 373.13 & 1.151 & \begin{tabular}{|l|}
16.4958222 \\
\end{tabular} & 82.908004 & \begin{tabular}{|l|}
1989,7921 \\
\end{tabular} \\
\hline & 18 & 2.24 & 100 & 31.67 & 373 & 1.144 & 6.4269137 & 32,301668 & 775.24004 \\
\hline & 19 & 1.95 & 99.91 & 30 & 372.91 & 1.149 & \begin{tabular}{|l|}
4.2594966 \\
\end{tabular} & 21.40823 & \begin{tabular}{|l|}
513.79751 \\
\end{tabular} \\
\hline & 20 & 2.22 & 99.95 & 28 & 372.95 & 1.157 & 6.3294115 & 31.811622 & 7633.47894 \\
\hline & 21 & 2.5 & 99.9 & 26 & 372.9 & 1.164 & 9.0949943 & \begin{tabular}{|l|}
45,711442 \\
\end{tabular} & 1097.0746 \\
\hline & 22 & 1.95 & 99.75 & 28 & 372.75 & 1.155 & \begin{tabular}{|l|l|}
4.2809322 \\
\end{tabular} & 21.515965 & 516.38317 \\
\hline
\end{tabular}

\begin{tabular}{|c|c|c|c|c|c|c|c|c|c|}
\hline & 23 & 1.95 & 99.55 & 28 & 372.55 & 1.152 & 4.2723489 & 21.472825 & 515.34781 \\
\hline & 24 & 3.06 & 99.3 & 28 & 372.3 & 1.149 & 16,467783 & 82.767078 & 1986,4099 \\
\hline & 25 & 5.37 & 99.89 & 28.88 & 372.89 & 1.153 & 89.268582 & 488.65389 & 10767.933 \\
\hline & 26 & 5.29 & 100.02 & 31 & 373.02 & 1.146 & 84.853232 & 426.47235 & 102355336 \\
\hline & 27 & 4.17 & 100.03 & 30 & 373.03 & 1.150 & 41.704595 & 209.6073 & 5030.5751 \\
\hline & 28 & 3.06 & 100.01 & 30 & 373.01 & 1.150 & 16,476053 & 82.808643 & 1987.4074 \\
\hline & 29 & 2.5 & 100.06 & 28 & 373.06 & 1.158 & 9.0490323 & 45.480436 & 1091.505 \\
\hline & 30 & 2.78 & 100.01 & 28 & 373.01 & 1.158 & 12.436536 & 62.506029 & 1500.1447 \\
\hline \multicolumn{10}{|l|}{ JULY } \\
\hline & 1 & 2.22 & 99.84 & 28 & 372.84 & 1.156 & 6.3224457 & 31.776612 & 762.63869 \\
\hline & 2 & 0.556 & 99.83 & 26 & 372.83 & 1.163 & 0.0999775 & 0.502487 & 12.059689 \\
\hline & 3 & 3.06 & 99.94 & 29 & 372.94 & 1.153 & 16.519039 & 83.024692 & 1992.5926 \\
\hline & 4 & 2.69 & 99.89 & 30 & 372.89 & 1.149 & 11.17955 & 56,18842 & 1348.5221 \\
\hline & 5 & 1.11 & 100.05 & 30 & 373,05 & 1.151 & 0.7867405 & 3.5541578 & 94.899786 \\
\hline & 6 & 139 & 100.28 & 30 & 373.28 & 1.153 & 1.548475 & 7.7865355 & 1867.78325 \\
\hline & 7 & 2.24 & 100.29 & 30 & 373.29 & 1.153 & 6.4810768 & 32.573892 & 781.7734 \\
\hline & 8 & 1.34 & 100.12 & 28.88 & 373.12 & 1,156 & 13902375 & 6.9873337 & 167,69601 \\
\hline & 9 & 1.11 & 99.89 & 30 & 372.89 & 1.149 & 0.7854823 & 3.9478343 & 94.748023 \\
\hline & 10 & 1.69 & 99.7 & 30 & 372.7 & 1.146 & 2.7669464 & 13.906673 & 333,76015 \\
\hline & 11 & 1.95 & 99.6 & 28 & 372.6 & 1.153 & 4.2749947 & 21.48361 & 515.60665 \\
\hline & 12 & 3.34 & 99.89 & 28 & 372.89 & 1.156 & 21.541851 & 108.26935 & 2598.4643 \\
\hline & 13 & 3.89 & 100.01 & 28 & 373.01 & 1.158 & 34,073272 & 171.1.2527 & 4110.0544 \\
\hline & 14 & 2.22 & 99.9 & 28 & 372.9 & 1.156 & 6.3262452 & 31.795709 & 763.09701 \\
\hline & 15 & 1,39 & 99.85 & 28 & 372.85 & 1.156 & 1.5520799 & 7.8007537 & 187.21809 \\
\hline & 16 & 1.95 & 99.79 & 26 & 372.79 & 1.163 & 4.3112954 & 21.66857 & 522.04569 \\
\hline & 17 & 2.22 & 99.81 & 28 & 372.81 & 1.155 & 6.3205459 & 31.,767064 & 762.40953 \\
\hline & 18 & 1.39 & 99.67 & 28 & 372.67 & 1.154 & 1.549282 & 7.7866913 & 186.88059 \\
\hline & 19 & 0.834 & 99.58 & 27 & 372.58 & 1.157 & 0.3354572 & 1.6860079 & 40.46419 \\
\hline & 20 & 1.11 & 99.74 & 27 & 372.74 & 1.158 & 0.7921459 & 3.9813251 & 95,551801 \\
\hline & 21 & 1,39 & 99.53 & 27 & 372.53 & 1.156 & 1.5522628 & 7.801673 & 187.24015 \\
\hline & 22 & 2.5 & 99.38 & 28 & 372.38 & 1.150 & 8.9875357 & 45.171355 & 1084,1125 \\
\hline & 23 & 1.95 & 99.51 & 27 & 372.51 & 1.156 & 4.2848677 & 21.535745 & 516.85788 \\
\hline & 24 & 1.95 & 99.7 & 26 & 372.7 & 1.162 & 4307407 & 21.649028 & 519.57666 \\
\hline & 25 & 2.5 & 99.62 & 27 & 372.62 & 1.157 & 9.0392712 & 45.431377 & 1090.353 \\
\hline & 26 & 4.45 & 99.64 & 26 & 372.64 & 1.161 & 51.160016 & 257.13024 & 6171.1258 \\
\hline & 27 & 4.73 & 100 & 28 & 373 & 1.158 & 61.249851 & 307.84175 & 7388.202 \\
\hline & 28 & 3.61 & 100.19 & 28 & 373.19 & 1.160 & 27,281459 & 137.11661 & 3290,7987 \\
\hline & 29 & 1.95 & 99.87 & 28 & 372.87 & 1.156 & 4.2860822 & 21.541849 & 517,00438 \\
\hline & 30 & 3.06 & 99.41 & 26 & 372.41 & 1.158 & 16.5963 & 83.413003 & 2001.9121 \\
\hline & 31 & 3.06 & 99.43 & 27 & 372.43 & 1.155 & 16.544307 & 83.151685 & 1995.6404 \\
\hline \multicolumn{10}{|l|}{$\mathrm{AUG}$} \\
\hline & 1 & 3.34 & 99.73 & 28 & 372.73 & 1.154 & 21.507346 & 108.09592 & 2594,3022 \\
\hline & 2 & 2.22 & 100.07 & 30 & 373.07 & 1.151 & 6.2951822 & 31.639586 & 759,35005 \\
\hline & 3 & 2.24 & 100.16 & 30 & 373.16 & 1.152 & 6.4726757 & 32.531668 & 780.76004 \\
\hline & 4 & 1.79 & 100.09 & 30 & 373.09 & 1.151 & 3,300618 & 16.588906 & 398.13374 \\
\hline & 5 & 1.11 & 100.06 & 30 & 373.06 & 1.151 & 0.7868191 & 3.954553 & 94.909272 \\
\hline
\end{tabular}


INTERNATIONAL JOURNAL OF INNOVATIVE RESEARCH IN ELECTRICAL, ELECTRONICS, INSTRUMENTATION AND CONTROL ENGINEERING Vol. 3, Issue 2, February 2015

\begin{tabular}{|c|c|c|c|c|c|c|c|c|c|}
\hline & 6 & 1.95 & 100.18 & 28 & 373.18 & 1.160 & 4.2993864 & 21.608716 & 518.60918 \\
\hline & 7 & 3.06 & 100.29 & 28 & 373.29 & 1.161 & 16.631963 & 83.592248 & 2006.214 \\
\hline & 8 & 1.95 & 100.18 & 28 & 373.18 & 1.160 & 4.2993864 & 21.608716 & 518.60918 \\
\hline & 9 & 0.834 & 100.21 & 30 & 373.21 & 1.152 & 0.3342371 & 1.6798758 & 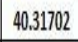 \\
\hline & 10 & 1.39 & 100.26 & 30 & 373.26 & 1.153 & 1.5481662 & 7.7810833 & 186.746 \\
\hline & 11 & 1.11 & 100.39 & 27 & 373.39 & 1.166 & 0.7973082 & 4.0072711 & 96.177507 \\
\hline & 12 & 1.39 & 100.27 & 29 & 373.27 & 1.157 & 1.5534775 & 7.8076271 & 187,38305 \\
\hline & 13 & 1.39 & 100.2 & 28 & 373.2 & 1.160 & 1.5752204 & 7.8280974 & 187.87734 \\
\hline & 14 & 1.39 & 100.2 & 29 & 373.2 & 1.156 & 1.552363 & 7.8021765 & 187,25224 \\
\hline & 15 & 1.67 & 100.23 & 30 & 373.23 & 1.153 & 2.6840625 & 13.490098 & 323.76235 \\
\hline & 16 & 1.95 & 100.08 & 30 & 373,08 & 1.151 & 4.2667442 & 21.,444657 & 514,67176 \\
\hline & 17 & 1.79 & 100,02 & 30 & 373,02 & 1.150 & 3.2983096 & 16.577304 & 397.8553 \\
\hline & 18 & 1.39 & 99.99 & 28 & 372.99 & 1.157 & 1.5542561 & 78116912 & 187,48059 \\
\hline & 19 & 3.13 & 99.79 & 27.78 & 372.79 & 1.156 & 17,723896 & \begin{tabular}{|l}
89.0803 \\
\end{tabular} & 2137.9272 \\
\hline & 20 & 3.33 & 99,61 & 27 & 372.61 & 1.157 & 21.360061 & 107,35567 & 2576.536 \\
\hline & 21 & 2.5 & 99,68 & 28 & 372.68 & 1.154 & 9.0146666 & 45,307714 & 1087,3851 \\
\hline & 22 & 2.5 & 99.92 & 32 & 372.92 & 1.141 & 8.9178614 & 44.821172 & 1075,7081 \\
\hline & 23 & 1.39 & 100.09 & 31 & 373.09 & 1.147 & 1.5404571 & 7.7423375 & 185.8161 \\
\hline & 24 & 1.67 & 100.19 & 30 & 373.19 & 1.152 & 2.6829913 & 13,487714 & 323.63315 \\
\hline & 25 & 2.22 & 100.14 & 30 & 373.14 & 1.152 & 6.2995857 & 31.661718 & 759.88123 \\
\hline & 26 & 1.39 & 100.11 & 30 & 373.11 & 1.151 & 1.54585 & 7.7694419 & 186.46661 \\
\hline & 27 & 1.39 & 100.18 & 28 & 373.18 & 1.160 & 1.5572095 & 7.8265349 & 187,.83584 \\
\hline & 28 & 5.28 & 100.41 & 26 & 373.41 & 1.170 & 86.118341 & 432.83078 & 10387.939 \\
\hline & 29 & 1.67 & 100.53 & 28 & 373.53 & 1.164 & 2.7099839 & $\mid 13.620379$ & \begin{tabular}{|l}
326.8891 \\
\end{tabular} \\
\hline & 30 & 2.78 & 100.49 & 29 & 373.49 & 1.159 & 12.454847 & 62.598061 & 1502,3535 \\
\hline & 31 & 2.5 & 100.42 & 30 & 373.42 & 1.155 & 9.0216448 & 45,342787 & 1088.2269 \\
\hline \multicolumn{10}{|l|}{ SETT } \\
\hline & 1 & 2.24 & 100.43 & 30.56 & 373.43 & 1.153 & 6.4781512 & 32.559188 & 781.42051 \\
\hline & 2 & 1.11 & 100.5 & 30 & 373.5 & 1.156 & 0.7902791 & 3.9719926 & 95.326622 \\
\hline & 3 & 2.5 & 100.5 & 28 & 373.5 & 1.163 & 9.0888241 & 45.68043 & 1096.3303 \\
\hline & 4 & 2.22 & 100.44 & 30 & 373.44 & 1.155 & 6.3184581 & 31.75657 & 762.15768 \\
\hline & 5 & 1.95 & 100.24 & 32 & 373.24 & 1.145 & 4.2455422 & 21.338095 & 512.11428 \\
\hline & 6 & 1.67 & 100,47 & 30 & 373.47 & 1.155 & 2.6904895 & 13.5224 & \begin{tabular}{|l}
324.5376 \\
\end{tabular} \\
\hline & 7 & 1.39 & 100.62 & 29 & 373.62 & 1.161 & 1.5586699 & 7.8348802 & 188.03713 \\
\hline & 8 & 1.39 & 100.68 & 27 & 373.68 & 1.169 & 1.5701981 & 7.8918159 & 189.40358 \\
\hline & 9 & 0.834 & 100.75 & 28 & 373.75 & 1.166 & 0.338271 & 1.7001502 & 40.803606 \\
\hline & 10 & 1.11 & 100.61 & 28 & 373.61 & 1.165 & 0.7964008 & 4.0027105 & 96.065052 \\
\hline & 11 & 1.11 & 100.61 & 30 & 373.61 & 1.157 & 0.791144 & 3.97629 & 95.43096 \\
\hline & 12 & 1.67 & 100.74 & 30 & 373.74 & 1.158 & 2.6977198 & 13.55874 & 325.40975 \\
\hline & 13 & 1.67 & 100.7 & 30 & 373.7 & 1.158 & 2.6966486 & 13.553356 & 325.28055 \\
\hline & 14 & 1.67 & 100.4 & 30 & 373.4 & 1.155 & 2.6886149 & 13.512979 & 32431149 \\
\hline & 15 & 0.834 & 100.23 & 30 & 373.23 & 1.153 & 0.33403038 & 1.6802111 & 40,325066 \\
\hline & 16 & 0.834 & 100.21 & 28 & 373.21 & 1.160 & 0.336458 & 1.6910378 & 40.584907 \\
\hline & 17 & 1.67 & 100.16 & 28 & 373.16 & 1.159 & 2.7000098 & 13.570249 & 325.68598 \\
\hline & 18 & 1.39 & 100.11 & 27 & 373.11 & 1.163 & 1.5613085 & 7.8471364 & 188.33127 \\
\hline & 19 & 2.22 & 99.94 & 28 & 372.94 & 1.157 & 6.3287783 & 31.80844 & 763.40255 \\
\hline
\end{tabular}

\begin{tabular}{|c|c|c|c|c|c|c|c|c|c|}
\hline & 20 & 2.22 & 100.03 & 27 & 373.03 & 1.162 & 6.3555925 & $31 ., 943208$ & 766.63699 \\
\hline & 21 & 1.67 & 100.12 & 28 & 373.12 & 1.159 & 2.6989315 & 13.56483 & 325.55592 \\
\hline & 22 & 1.95 & 100.16 & 28 & 373.16 & 1.159 & 4.298528 & 21,604402 & 518.50564 \\
\hline & 23 & 1.11 & 100.25 & 28 & 373.25 & 1.160 & 0.7935512 & 3,9883881 & 95.721315 \\
\hline & 24 & 1.39 & 100.43 & 30 & 373.43 & 1.155 & 1.5507913 & 7,7942768 & 187,06264 \\
\hline & 25 & 1.67 & 100.63 & 30 & 373.63 & 1.157 & 2.6947741 & 13.543935 & 325.05443 \\
\hline & 26 & 1.67 & 100.7 & 30 & 373.7 & 1.158 & 2.6966486 & 13.553356 & 325.28055 \\
\hline & 27 & 1.39 & 100.64 & 28 & 373.64 & 1.165 & 1.5645598 & 7.8624722 & 188.69933 \\
\hline & 28 & 1.39 & 100.47 & 27 & 373.47 & 1.167 & 1.566923 & 7.875355 & 189.00852 \\
\hline & 29 & 1.34 & 100.12 & 26.67 & 373.12 & 1.164 & 1.4004902 & 7,0388638 & 168.93273 \\
\hline & 30 & 0.56 & 100.07 & 28 & 373,07 & 1.158 & 0.1017161 & 0.511225 & 12.2694 \\
\hline \multicolumn{10}{|l|}{$O C T$} \\
\hline & 1 & 1.11 & 100.29 & 26 & 373.29 & 1.169 & 0.7991779 & 4.0166683 & 96,40004 \\
\hline & 2 & 1.11 & 100.63 & 27 & 373.63 & 1.169 & 0.7992143 & 4,0168512 & 96,404229 \\
\hline & 3 & 1.95 & 100.76 & 28 & 373.76 & 1.166 & 4.324278 & 21,733821 & 521.61171 \\
\hline & 4 & 2.5 & 100.71 & 28 & 373.71 & 1.166 & 9,1078157 & 45,775882 & 1098.6212 \\
\hline & 5 & 2.22 & 100.61 & 30 & 373.61 & 1.157 & 63291524 & 31.81032 & 763.47768 \\
\hline & 6 & 1.95 & 100,64 & 30 & 373.64 & 1.157 & 4.2906189 & 21.564651 & 517.55161 \\
\hline & 7 & 0.834 & 100.76 & 30 & 373.76 & 1.159 & 0,3360716 & 1.6890958 & 40.538298 \\
\hline & 8 & 1.39 & 100.85 & 28 & 373.85 & 1.167 & 1.567624 & 7.8788784 & 189.09308 \\
\hline & 9 & 1.39 & 100.85 & 28 & 373.85 & 1.167 & 1.567624 & 7.8788784 & 189.09308 \\
\hline & 10 & 1.67 & 100.9 & 28 & 373.9 & 1.168 & 2.719958 & 13,670509 & 328.09221 \\
\hline & 11 & 3.06 & 100.61 & 27 & 373.61 & 1.169 & 16.740649 & 84,1385 & 2019,324 \\
\hline & 12 & 6.67 & 99.59 & 24 & 372.59 & 1.168 & 173.35042 & 871.2592 & 20910.221 \\
\hline & 13 & 8.9 & 99.87 & 26 & 372.87 & 1.164 & 410.22487 & 2061.7902 & 49482.965 \\
\hline & 14 & 3.89 & 100.46 & 29 & 373.46 & 1.159 & 34.113254 & 171.45321 & 4114.8771 \\
\hline & 15 & 0.834 & 100.8 & 28 & 373.8 & 1.167 & 0.3384389 & 1.700994 & 40.823856 \\
\hline & 16 & 0.834 & 100.8 & 28 & 373.8 & 1.167 & 0.3384389 & 1.700994 & 40.823856 \\
\hline & 17 & 0.56 & 100.94 & 28 & 373.94 & 1.168 & 0.1026004 & 0.5156695 & 12,376069 \\
\hline & 18 & 0.834 & 101 & 28 & 374 & 1.169 & 0.3391104 & 1.704369 & 40.904856 \\
\hline & 19 & 0.834 & 101.05 & 28 & 374,05 & 1.170 & 0.3392783 & 1.7052127 & 40.925106 \\
\hline & 20 & 0.834 & 100.98 & 29 & 373.98 & 1.165 & 0.3379206 & 1.698389 & 40.761336 \\
\hline & 21 & 1.39 & 100.88 & 27 & 373.88 & 1.172 & 1.5733173 & 7.9074929 & 189.77983 \\
\hline & 22 & 2.22 & 100.99 & 24 & 373.99 & 1.185 & 6.4814019 & 32.575526 & 781.81263 \\
\hline & 23 & 3.06 & 101.07 & 24 & 374,07 & 1,186 & 16.987059 & 85.37696 & \begin{tabular}{|l|l}
2049.047 \\
\end{tabular} \\
\hline & 24 & 2.22 & 101 & 25 & 374 & 1.181 & 6.4602919 & 32.469427 & 779.26625 \\
\hline & 25 & 2.22 & 100.87 & 24 & 373.87 & 1.183 & 6.4737005 & 32.536819 & 780.88365 \\
\hline & 26 & 1.11 & 100.9 & 25 & 373.9 & 1.180 & 0.8067369 & 4.0546599 & 97.311837 \\
\hline & 27 & 1.67 & 100.95 & 23 & 373.95 & 1.188 & 2.7672738 & 13.908318 & 333.79964 \\
\hline & 28 & 1.39 & 100.95 & 26 & 373.95 & 1.176 & 1.5796746 & 7.9394447 & 190.54667 \\
\hline & 29 & 1.11 & 101.19 & 26 & 374.19 & 1.179 & 0.8063497 & 4.0527138 & 97.265131 \\
\hline & 30 & 6.12 & 101.33 & 28 & 374,33 & 1.173 & 134,43549 & 675.67275 & 16216.146 \\
\hline & 31 & 1.39 & 101.41 & 24 & 374,41 & 1.190 & 1.5975588 & 8.0293303 & 192.70393 \\
\hline \multicolumn{10}{|l|}{ Nov } \\
\hline & 1 & 1.11 & 101.4 & 26 & 374.4 & 1.182 & 0.8080232 & 4.0611244 & 97.466986 \\
\hline & 2 & 0.56 & 101.36 & 26 & 374,36 & 1.181 & $|0.1037164|$ & 0.5212788 & 12.510 \\
\hline
\end{tabular}


INTERNATIONAL JOURNAL OFINNOVATIVE RESEARCH IN ELECTRICAL, ELECTRONICS, INSTRUMENTATION AND CONTROL ENGINEERING Vol. 3, Issue 2, February 2015

\begin{tabular}{|c|c|c|c|c|c|c|c|c|}
\hline 3 & 0.834 & 101.47 & 25 & 374.47 & 1.186 & 0.3441182 & 1.7295381 & 41.508915 \\
\hline 4 & 0.834 & 101.59 & 24 & 374.59 & 1.192 & 0.3456852 & 1.7374138 & 41.69793 \\
\hline 5 & 1.11 & 101.59 & 24 & 374.59 & 1.192 & 0.8149886 & 4,0961329 & 98.30719 \\
\hline 6 & 1.11 & 101.54 & 25 & 374.54 & 1.187 & 0.811854 & 4,0803782 & 97.929078 \\
\hline 7 & 1.11 & 101.4 & 24 & 374.4 & 1.190 & 0.8134644 & 4,0884721 & 98.123329 \\
\hline 8 & 1.11 & 101.3 & 24 & 374.3 & 1.188 & 0.8126622 & 4.08444 & 98.026561 \\
\hline 9 & 1.11 & 101.26 & 24 & 374.26 & 1.188 & 0.8123413 & 4,0828272 & 97.987853 \\
\hline 10 & 1.11 & 101.23 & 24 & 374.23 & 1.188 & 0.8121006 & 4,0816176 & 97.958823 \\
\hline 11 & 1.67 & 101.11 & 24 & 374,11 & 1.186 & 2.7623276 & 13.883458 & 333.203 \\
\hline 12 & 1.34 & 101.21 & 22.78 & 374.21 & 1.192 & 1.4343566 & 7.2090761 & 173.01783 \\
\hline 13 & 1.67 & 101.21 & 23 & 374.21 & 1.191 & 2.774401 & 13.94414 & 334.65935 \\
\hline 14 & 1.39 & 101.3 & 22 & 374.3 & 1.196 & 1.606645 & 8.074998 & 193.79995 \\
\hline 15 & 1.11 & 101.34 & 22 & 374.34 & 1.197 & 0.8184948 & 4.1137549 & 98.730118 \\
\hline 16 & 1.11 & 101.43 & 22 & 374.43 & 1.198 & 0.8192217 & 4.1174083 & 98.8178 \\
\hline 17 & 1.67 & 101.4 & 24 & 374.4 & 1.190 & 2.7702504 & 13.923279 & 334.15868 \\
\hline 18 & 1.39 & 101.43 & 24 & 374.43 & 1.190 & 1.5978738 & 8.0309139 & 192.74193 \\
\hline 19 & 1.95 & 101.38 & 22 & 374.38 & 1.197 & 4,4393789 & 22.312318 & 535.49564 \\
\hline 20 & 1.79 & 101.21 & 23.33 & 374.21 & 1.190 & 3.4126756 & 17.152107 & 411.65058 \\
\hline 21 & 2.22 & 101.14 & 22 & 374,14 & 1.195 & 6.5350357 & 32.845089 & 788.28215 \\
\hline 22 & 1.95 & 101.31 & 25 & 374.31 & 1.185 & 4.3916528 & 22.072447 & 529.73872 \\
\hline 23 & 0.278 & 101.34 & 25 & 374,34 & 1.185 & 0.0127288 & 0.0639749 & 1.5353976 \\
\hline 24 & 1.11 & 101.26 & 26 & 374.26 & 1.180 & 0.8069075 & 4.0555173 & 97.332416 \\
\hline 25 & 1.39 & 101.08 & 24 & 374,08 & 1.186 & 1.5923601 & 8.003202 & 192.07685 \\
\hline 18 & 0.834 & 101.26 & 22 & 374.26 & 1.196 & 0,3468983 & 1.7435108 & 41.84426 \\
\hline 19 & 1.11 & 101.31 & 22 & 374,31 & 1.197 & 0.8182525 & 4.1125371 & 98.70089 \\
\hline 20 & 1.39 & 101.46 & 23 & 374.46 & 1.194 & 1.6037463 & 8,0604287 & 193.45029 \\
\hline 21 & 1.11 & 101.58 & 22 & 374.58 & 1.200 & 0,8204332 & 4.1234974 & 98.963937 \\
\hline 22 & 1.11 & 101.73 & 22 & 374.73 & 1.202 & 0.8216447 & 4.1295864 & 99.110074 \\
\hline 23 & 0.834 & 101.76 & 22 & 374.76 & 1.202 & 0,3486112 & 1.7521199 & 42.050878 \\
\hline 24 & 1.34 & 101.78 & 21.11 & 374.78 & 1.206 & 1.450625 & 7.2908413 & 174.98019 \\
\hline 25 & 0.834 & 101.78 & 23 & 374.78 & 1.198 & 0.3475017 & 1.7465438 & 41.917051 \\
\hline 26 & 0.834 & 101.68 & 22 & 374.68 & 1.201 & 0,3483371 & 1.7507425 & 42,017819 \\
\hline 27 & 0.9 & 101.61 & 21.67 & 374,61 & 1.201 & 0.4379416 & 2.2010943 & 52.826263 \\
\hline 28 & 0.834 & 101.58 & 22 & 374.58 & 1.200 & 0.3479946 & 1.7490206 & 41,976496 \\
\hline 29 & 0.834 & 101.55 & 22 & 374.55 & 1.199 & 0,3478918 & 1,7485041 & 41.964098 \\
\hline 30 & 1.67 & 101.7 & 22 & 374.7 & 1.201 & 2,7972833 & 14,059146 & 337,4195 \\
\hline 31 & 1.39 & 101.76 & 22 & 374.76 & 1.202 & 1.6139408 & 8.1116663 & 194,67999 \\
\hline
\end{tabular}

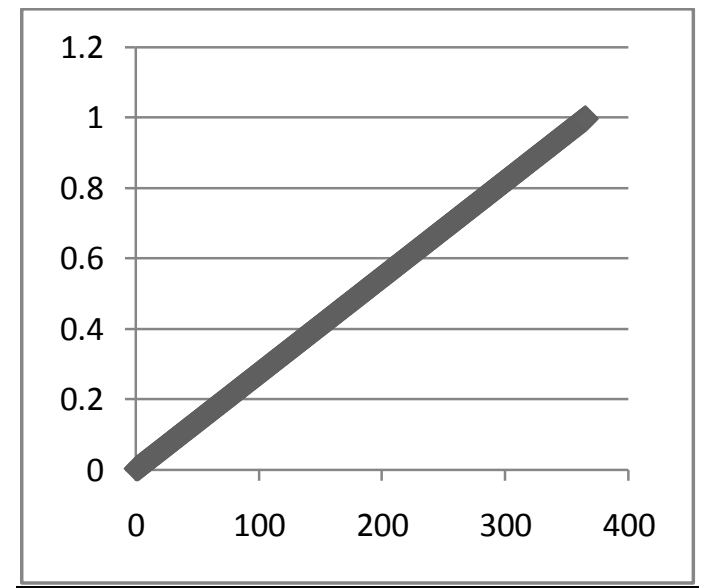

Graph between Speed(m/sec) \& Rate of return:

It includes in Y-axis the daily speed in descending order and in $\mathrm{X}$-axis the rate of return.

Rate of return= 1/Probability $(\mathrm{F}(\mathrm{U}))$

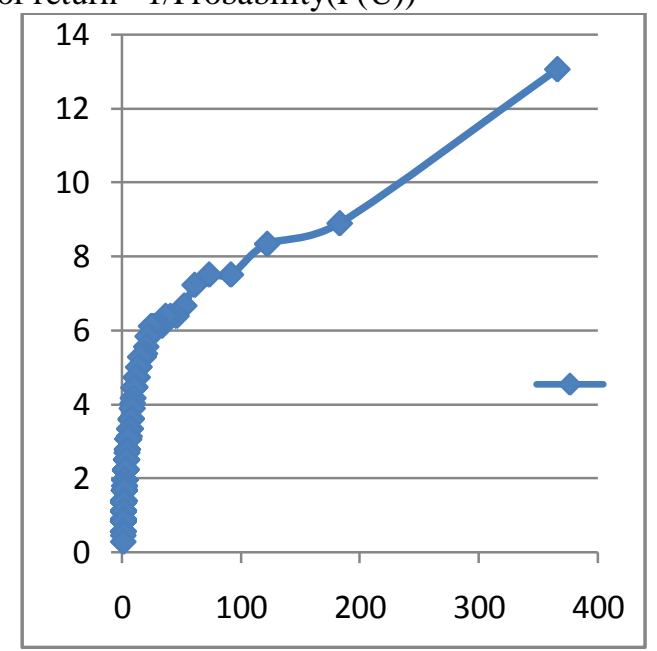

Histogram \& Approximate graph between Freq. \& Energy for 1 year:

Wind velocity are assigned to ' $\mathrm{k}$ ' equally distribution classes of width $\Delta \mathrm{v}=1 \mathrm{~m} / \mathrm{sec}$ with centre values of $\operatorname{vi}(\mathrm{i}=1 \ldots \mathrm{k})$.

$\mathrm{hi}=$ Relative frequency of wind velocity in the period

\section{RESULTS OBTAINED:}

Graph between Speed $(\mathrm{m} / \mathrm{sec}) \&$ Probability, F(U): under consideration and is equal to $\mathrm{hi}=\mathrm{ti} / \mathrm{T} ; \mathrm{T}=365$ days

It includes in Y-axis the daily speed in descending order The graph includes 'hi' in X-axis \& $\operatorname{speed}(\mathrm{m} / \mathrm{sec})$ in $\mathrm{Y}$ and in $\mathrm{X}$-axis the probability obtained from equation axis.

$F(U)=\operatorname{speed} /(N+1)$, where $N=$ No. of days. For yearly calculation, $\mathrm{N}=365$. 


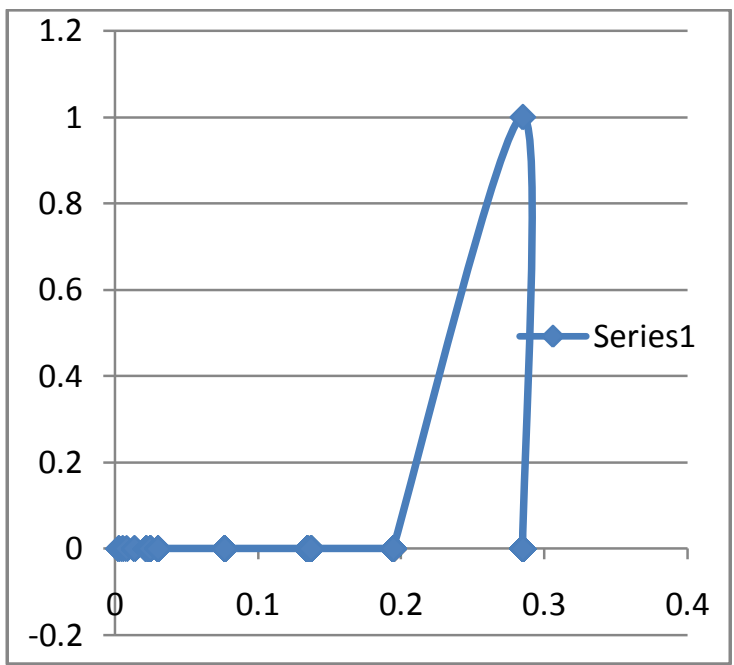

E. DIFFERENT PARAMETERS CALCULATION:

The average wind speed $\&$ average power from the yearly data of 2013 is obtained as $2.197 \mathrm{~m} / \mathrm{sec} \& 6.617 \mathrm{KW}$ respectively.

TABLE II

RELATION BETWEEN ROTOR DIAMETER, ROTOR SPEED \& RATED POWER

\begin{tabular}{|c|c|c|c|c|c|c|c|c|c|c|c|c|}
\hline SL.NO & DEATURES & \multicolumn{2}{|c|}{ SMALL } & \multicolumn{3}{|c|}{ MEDIUM } & \multicolumn{3}{|c|}{ LARGE } & \multicolumn{3}{|c|}{ VERYLARGE } \\
\hline 1 & RATED POWER(KW) & 10 & 25 & 50 & 100 & 150 & 250 & 500 & 1000 & 2000 & 3000 & 4000 \\
\hline 2 & ROTOR DIAMEER(M) & 64 & 10 & 14 & 20 & 25 & 32 & 49 & 64 & 90 & 110 & 130 \\
\hline & & & & & & & & & & & & \\
\hline 3 & ROTOR SPEED(RPM) & 200 & 150 & 100 & 67 & 55 & 43 & 29 & 19 & 15 & 13 & 11 \\
\hline
\end{tabular}

In order to get suitable rotor speed in Bhubaneswar whose average wind speed is $2.197 \mathrm{~m} / \mathrm{sec}$, the rotor diameter is considered to be $6.4 \mathrm{~m}$ which comes in the range of small wind turbine. Hence, the rated power we can get is $10 \mathrm{KW}$. The maximum torque developed on any ideal turbine would occur if maximum circumferential force acts on the tip of blade with radius $\mathrm{R}$.

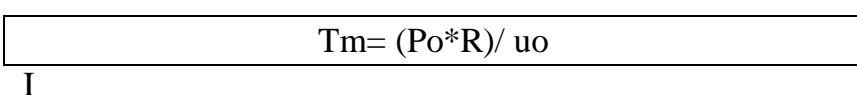

$\mathrm{n}$ this case, $\mathrm{Tm}=(6.617 * 6.4) / 2.197=19.276 \mathrm{Nm}$.

Practical Observations indicates Tip Speed ratio, TSR= $\left(4 \prod / n\right)$, where $n=$ No. of blades.

For a 3-bladed turbine, $\mathrm{TSR}=(4 * 3.14 / 3)=4.186$.

For a practical machine where circumferential force is not concentrated at the tip but spread through the length of the blade, less shaft torque will be produced which is given by

\section{$\mathrm{Tsh}=\mathrm{CT} * \mathrm{Tm}$}

where $\mathrm{CT}=$ Torque Coefficient $\&$ is gievn by $\mathrm{CT}=\mathrm{Cp} / \mathrm{TSR}$. Maximum $\mathrm{CT}=\mathrm{Cpmax} / \mathrm{TSR}$ $=0.593 / 4.186=0.142$

Hence, $\mathrm{Tsh}=0.142 * 19.276=2.737 \mathrm{Nm}$

This can be used as an input to various types of generators simulation to get the desired output parameters like voltages \& current.

\section{F. SIMULATION WITH DIFFERENT GENERATORS:}

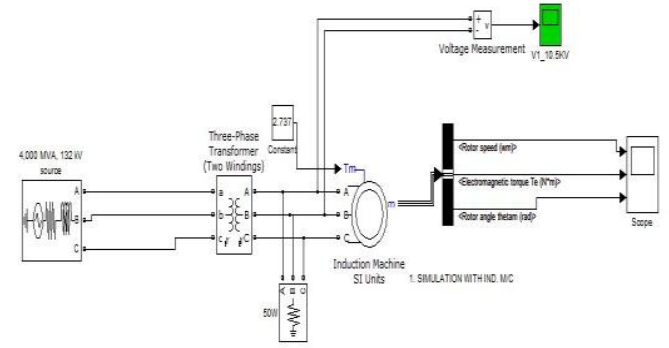

(Fig.1. SIMULATION WITH IND. GENERATOR)

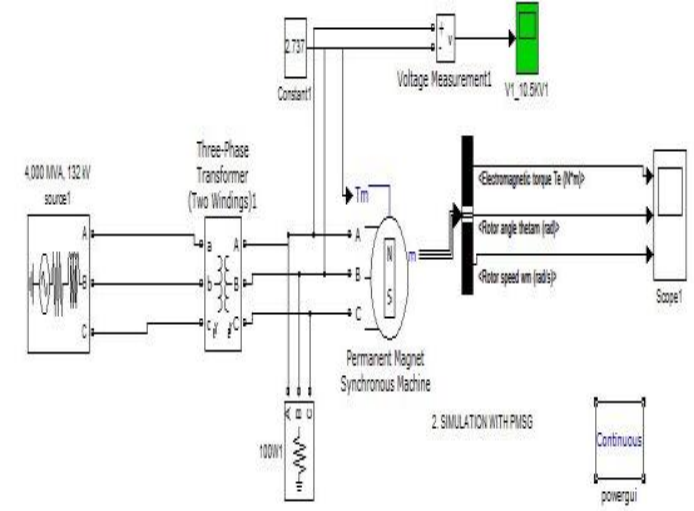

(Fig.2. SIMULATION WITH SYN. GENERATOR)

\section{G. RESULTS OBTAINED WITH DIFFERENT} GENERATORS:

The result includes the Voltage generated \& Other parameters like electromagnetic torque $(\mathrm{Nm})$, rotor speed(rad/sec), rotor angle(rad).

SIMULATION WITH IND. GENERATOR

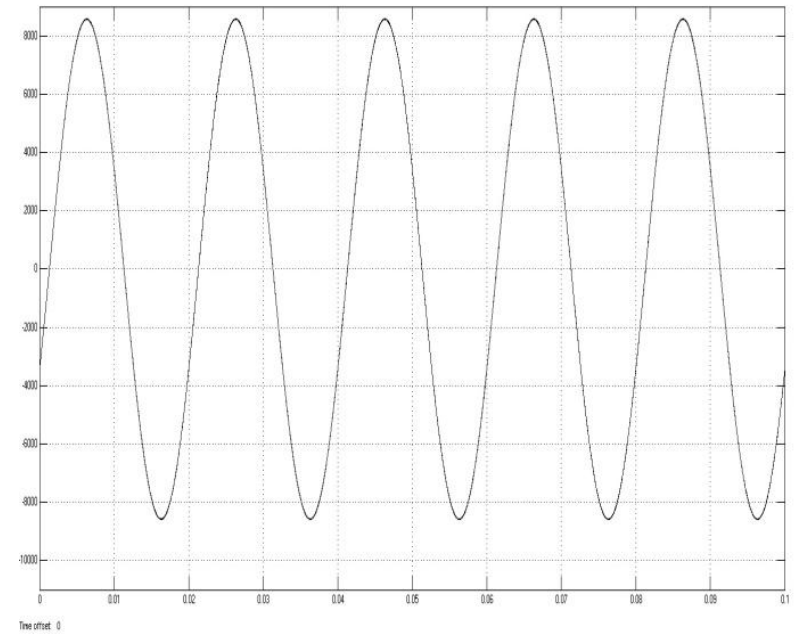

(Fig.3. Obtained Voltage) 


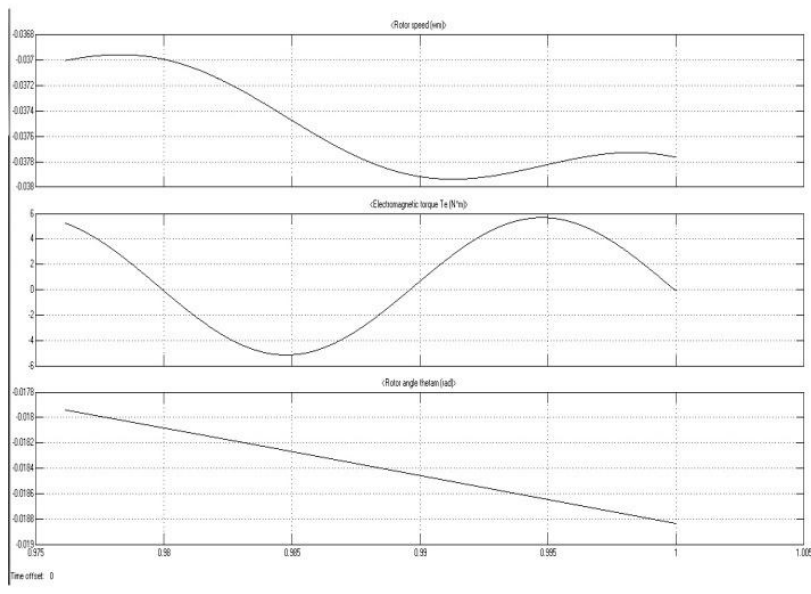

(Fig.4. Other Parameters)

\section{SIMULATION WITH SYN. GENERATOR}

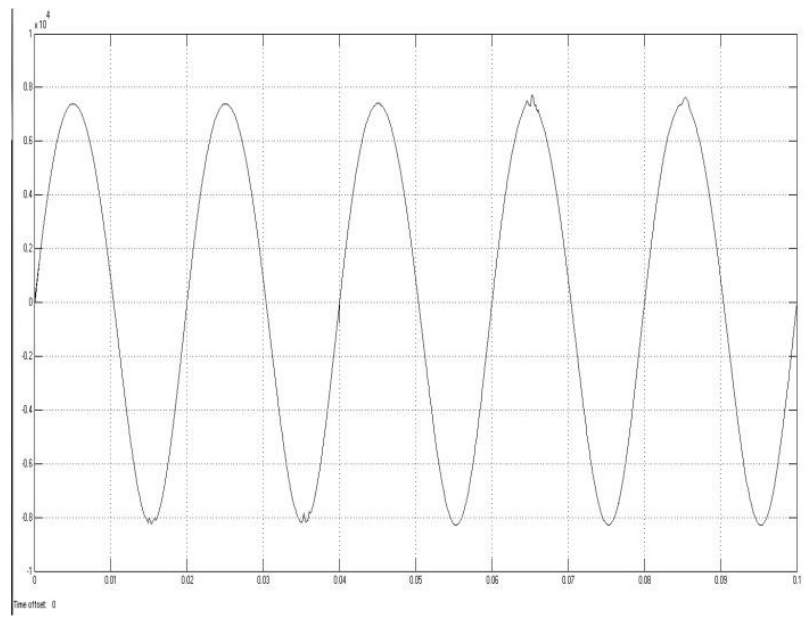

(Fig.5. Obtained Voltage)

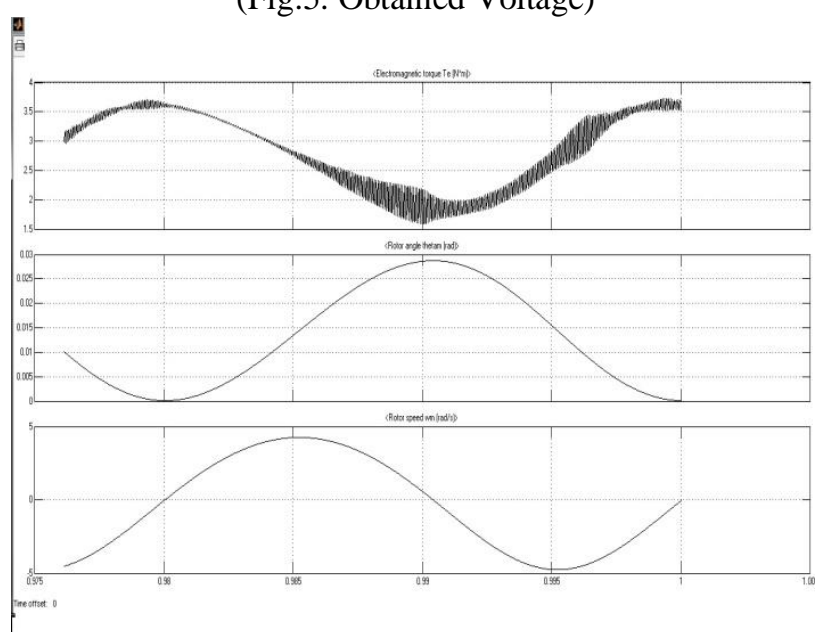

(Fig.6. Other Parameters)

\section{WIND ENERGY AND THE ENVIRONMENT} A. POSITIVE BENIFITS OF WIND ENERGY

It doesn't involves combustion or nuclear reaction, it is pollution free. It is renewable and plentiful. It can be installed in remote areas, in mountains and coastal regions.
It omits the release of GHG associated with fossil fuels. The general equation for estimating the reduction in emitted gas is:

Gas emission reduction(in tonnes) $=\mathrm{A} * 0.8 * \mathrm{~h} * \mathrm{KG}$

where $\mathrm{A}=$ rated capacity of development in $\mathrm{KW}, \mathrm{h}=$ No. of operational hrs per year $=8760 \mathrm{hrs}, \mathrm{KG}=$ specific gas constant.

$\mathrm{A}=10 \mathrm{KW}$;

$\mathrm{CO} 2$ emission reduction (in tonnes) $=$ $10 * 0.8 * 8760 * 862 / 1000000=60.4$

$\mathrm{SO} 2$ emission reduction (in tonnes) $=$ $10 * 0.8 * 8760 * 9.9 / 1000000=0.693$

$\mathrm{NO} 2$ emission reduction $($ in tonnes $)=$ $10 * 0.8 * 8760 * 2.8125 / 1000000=0.0197$

\section{B. NEGATIVE IMPACTS OF WIND ENERGY} NOISE:

The moving parts in wind turbine creates noise. Well developed technology and well designed wind turbines are generally quiet in operation compared to noise of road traffic, trains, aircrafts etc.

TABLE III

DIFF. SOURCES \& THEIR NOISE LEVELS IN DB

\begin{tabular}{|l|c|}
\hline \multicolumn{1}{|c|}{ SOURCE/ACTIVITY } & $\begin{array}{c}\text { INDICATIVE } \\
\text { NOISE } \\
\text { LEVELdB(A) }\end{array}$ \\
\hline Threshold of hearing & 0 \\
\hline Rural night time background & $\mathbf{2 0 - 4 0}$ \\
\hline Wind farm at 300m & $35-45$ \\
\hline Car at 40mph at $100 \mathrm{~m}$ & 55 \\
\hline Busy general office & 60 \\
\hline Truck at 30mph at $100 \mathrm{~m}$ & 65 \\
\hline Pneumatic drill at $7 \mathrm{~m}$ & 95 \\
\hline Jet air craft at $200 \mathrm{~m}$ & $\mathbf{1 0 5}$ \\
\hline Threshold of pain & $\mathbf{1 4 0}$ \\
\hline
\end{tabular}

There are two potential sources of noise related to wind turbines; the turbine blades passing through the air as the hub rotates and the gearbox and the generator. Noise from the blades is minimised by careful attention to the design and manufacturer of the blades. The noise from the gearbox and generator is controlled using the sound insulation and isolation materials.

OTHER FACTORS

Other factors which are associated with wind energy are Bird kill, Visual impacts, Shadow flicker \& communication interference.

A number of national wind energy associations have established detailed best practiced guidelines for development of wind farms including visual impacts.

\section{CONCLUSION}

In this paper, a detailed analysis of wind energy parameters at a specified location are presented and different constraints affecting it are figured. Then, an yearly data in that location is obtained and various calculations are done. Then, it is simulated \& results are studied. Its affect on the environment is also taken into account. 


\section{ACKNOWLEDGEMENT}

I have the greatest pleasure to offer my profound respect and pleasure to all members of Department of Electrical Engineering \& colleagues, KIIT University for their help in various ways for the completion of this paper.

\section{REFERENCES}

11] P.S Dokopoulos, A.C Saramourtsis, A.G Bakirtzis, "PREDICTION AND EVALUATION OF THE PERFORMANCE OF WINDDIESEL ENERGY SYSTEMS", IEEE Transactions on Energy Conversion, Vol.11, No.2, June (1996).

[2] Anders Grauers, "EFFICIENCY OF THREE WIND ENERGY GENERATOR SYSTEMS", "IEEE Transactions on Energy Conversion, Vol.11, No.3, September (1996).

[3] S.H. Karaki, R.B Chedid, R. Ramadan, "PROBABILISTIC PERFORMANCE ASSESSMENT OF AUTONOMOUS SOLARWIND ENERGY CONVERSION SYSTEM", "IEEE Transactions on Energy Conversion ,Vol.14, No.3, September (1999).

[4] Rajesh Karki, "RELIABILITY/COST IMPLICATIONS OF PV AND WIND ENERGY UTILIZATION IN SMALL ISOLATED POWER SYSTEMS", IEEE Transactions on Energy Conservation, Vol.16, No.4, December (2001).

[5] S.H Karaki, A. Salim, R.B Chedid," PROBABILISTIC MODEL OF A TWO-SITE WIND ENERGY CONVERSION SYSTEM","IEEE Transactions on energy conservation,Vol.17, No.4, December (2002). 